Supporting Information

\title{
Achieving High Conductivity at Low Ion Exchange Capacity for Anion Exchange Membranes with Electrospun Polyelectrolyte Nanofibers
}

Hanzhao Duan ${ }^{\dagger}$, Xia Cheng ${ }^{\dagger}$, Lingping Zeng ${ }^{\dagger}$, Qiang Liao ${ }^{\ddagger}$, Jianchuan Wang*, ${ }^{*}$ and Zidong Wei*, ${ }^{\dagger}$

' School of Chemistry and Chemical Engineering, Chongqing University, 400044, Chongqing, China

* School of Energy and Power Engineering, Chongqing University, 400044, Chongqing, China

E-mail addresses of Corresponding authors:

jxw319@cqu.edu.cn (J. Wang)

zdwei@cqu.edu.cn (Z. Wei) 


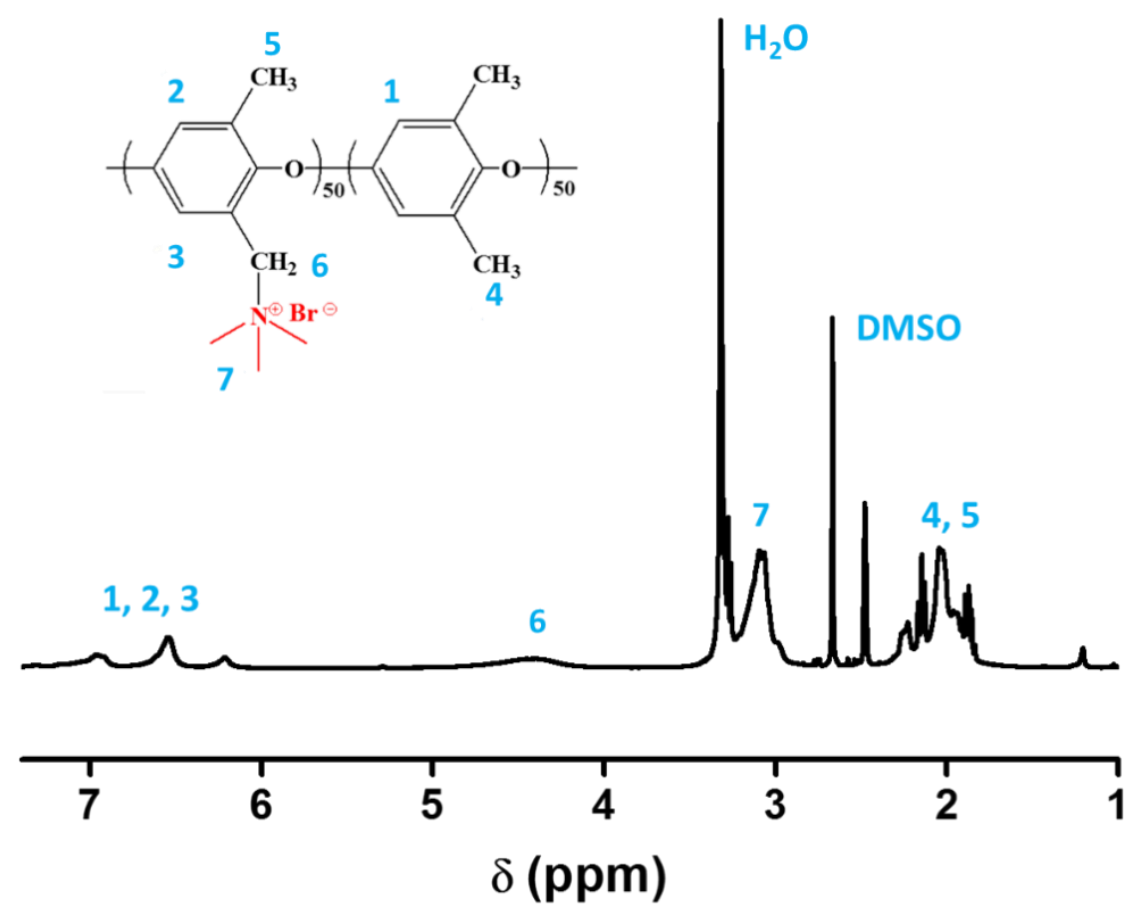

Figure S1. ${ }^{1} \mathrm{H}$ NMR spectrum of QPPO.

The chemical structure of QPPO was analyzed by the ${ }^{1} \mathrm{H}$ NMR spectrum. As shown in Figure S1, there were a chemical shift of methylene group at position 6 (4.3 ppm) and a chemical shift of methyl on quaternary ammonium groups $(3.0 \mathrm{ppm})$ at position 7 , which verified the successful synthesis of QPPO. A comparison of the integrals of the methylene groups (4.3 ppm) and the non-functionalized methyl groups (2.0 ppm) gave the quaternization degree (\%) of QPPO, which was determined to be about 50\%. 


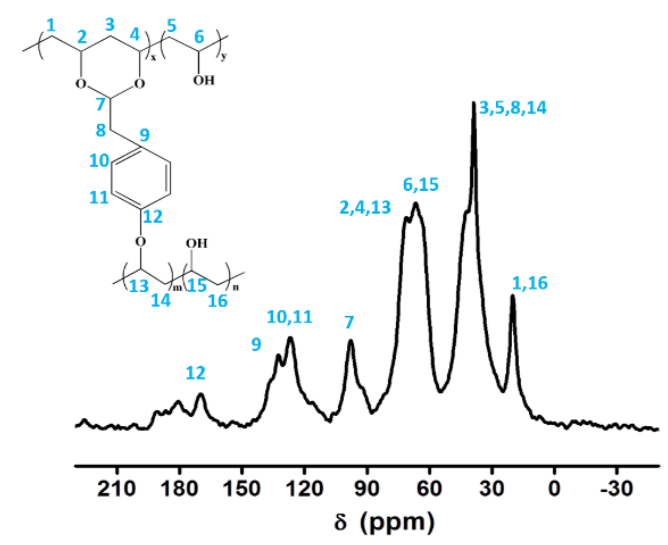

(a)

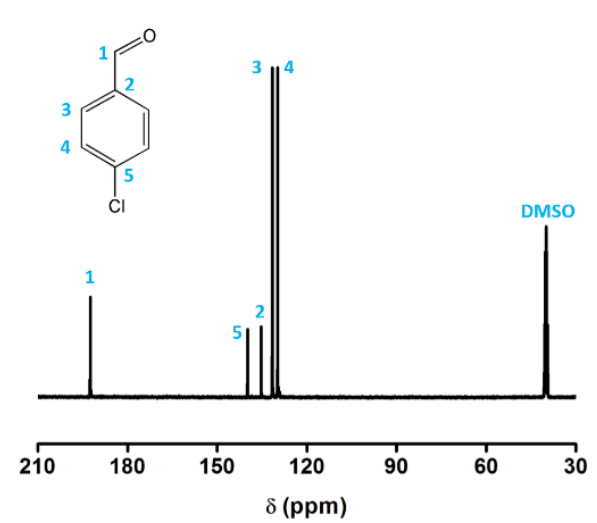

(b)

Figure S2. (a) Solid-state ${ }^{13} \mathrm{C}$ NMR spectra of the resultant of PVA reacting with 30 wt \% PCAD, (b) ${ }^{13} \mathrm{C}$ NMR spectra of PCAD.

In order to study the chemical structure of the resultant of PVA reacting with $30 \mathrm{wt} \%$ PCAD, the solid-state ${ }^{13} \mathrm{C}$ NMR was carried out. As shown in Figure S2 (a), the chemical shifts at $\delta=20.3-42.6$ ppm were mainly attributed to the $\mathrm{CH}_{2}-\mathrm{CH}_{2}$ (position 1,16 and position $3,5,14$ ) on the mainchain of PVA, which overlapped with the benzyl carbon at position 8 . The chemical shifts at $\delta=62.5 \mathrm{ppm}$ (position 6,15 ) were related to the carbons attached to the hydroxyl group on the PVA chain, and the chemical shifts at $\delta=71.4 \mathrm{ppm}$ can be attributed the $\mathrm{C}-\mathrm{O}$ bond at position $2,4,13$. The chemical shift at position 7 ( $\delta=97.8 \mathrm{ppm})$ indicated the successful acetalation of PVA and PCAD. The chemical shifts at $\delta=126.7-132.5 \mathrm{ppm}$ (position 9, 10,11) were ascribed to the carbons on benzene ring. The chemical shift of the carbon connecting to chloride atom on PCAD was at $\delta=139.6 \mathrm{ppm}$ (as shown in Figure S2 (b)), while a new chemical shift at position $12(\delta=169.8 \mathrm{ppm})$ was generated for the resultant of PVA reacting with PCAD, indicating a reaction between the chlorine of PCAD and the hydroxyl group of PVA. Above all, the chemical shifts at $\delta=97.8 \mathrm{ppm}$ and $\delta=169.8 \mathrm{ppm}$ verified that the PCAD was a cross-linker to the PVA, and the final membranes were cross-linked. 


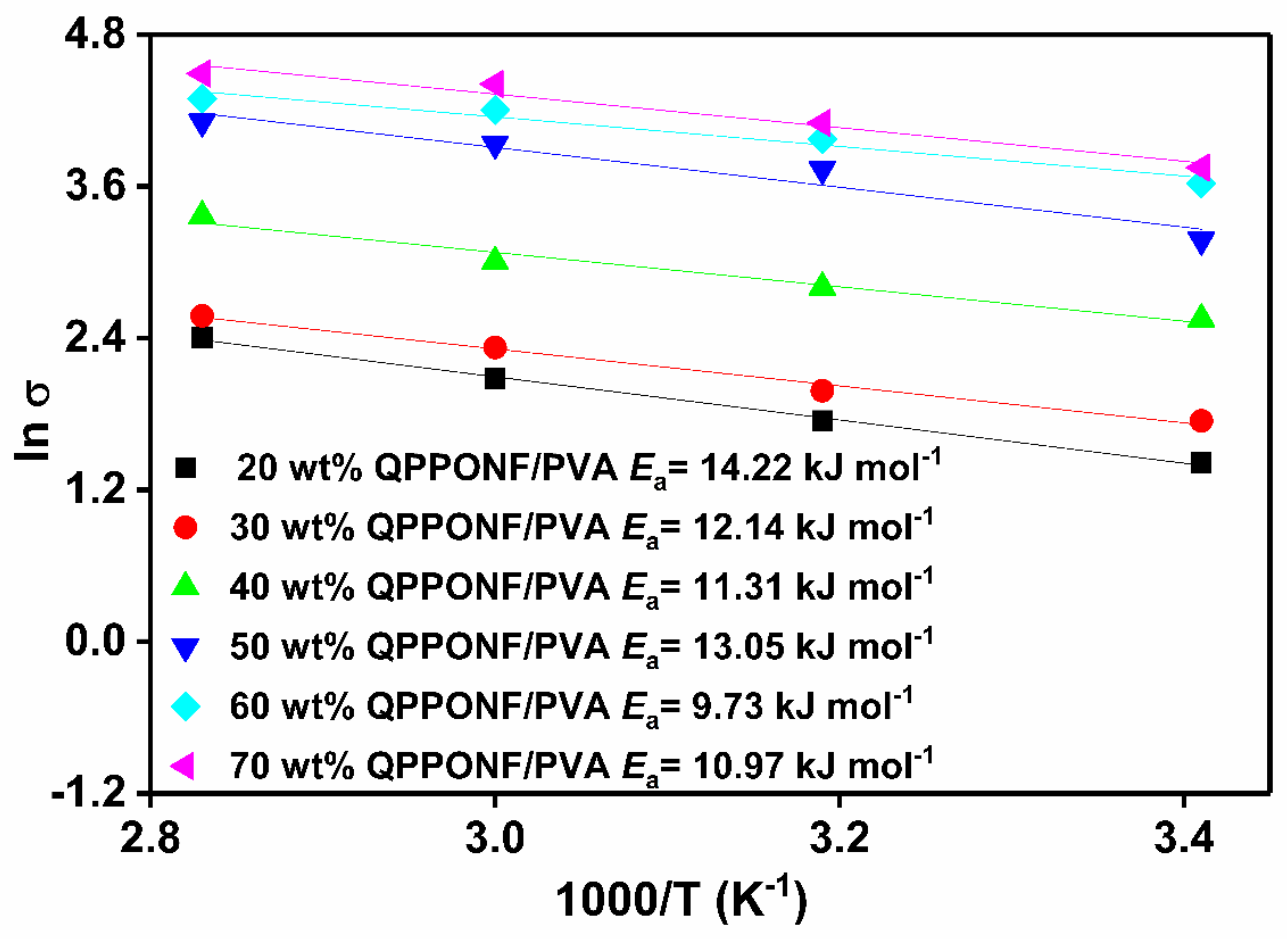

Figure S3. Arrhenius plot of the data in Figure 3 for the calculation of apparent activation energy $(E a)$.

In general, as the loading of QPPONF increased, the value of $E_{\mathrm{a}}$ showed a trend of decrease. The $E$ a of all samples were all in the range of $9.73-14.22 \mathrm{~kJ} \mathrm{~mol}^{-1}$. It was lower compared with quaternary ammonium type AEMs. ${ }^{1}$ 


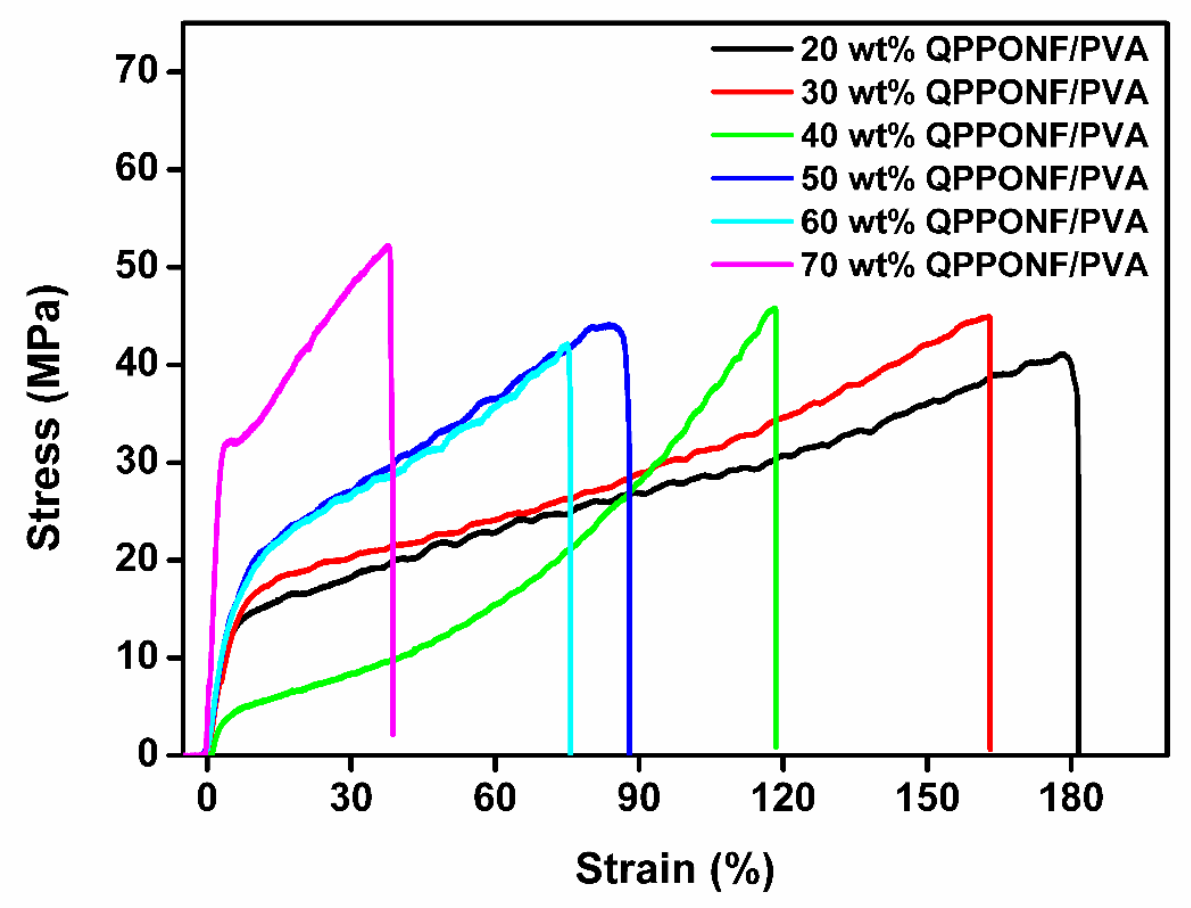

Figu re S4. Stress-Strain curves of dry composite membranes

As shown in Figure S4, with the loading of QPPONF increasing, the tensile strength showed a tendency of increase. The highest strength of $53 \mathrm{MPa}$ was obtained with 70 wt \% QPPONF incorporated. Compared with membranes at fully hydrated state, there was no water molecules between the polymer chains, thus the intermolecular forces between the polymers were larger. As a result, the tensile strength of dry membranes was almost twice, and the elongation increased as well. 


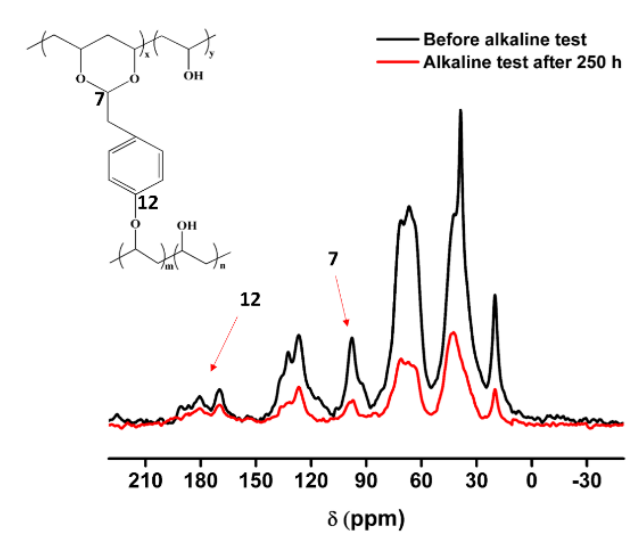

(a)

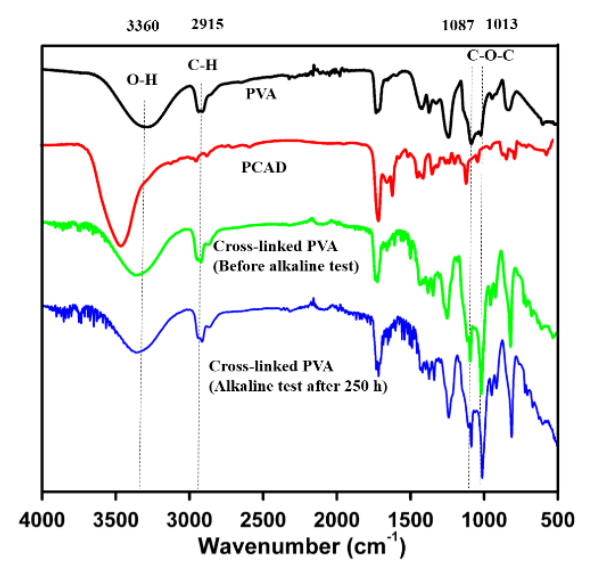

(b)

Figure S5. (a) Solid-state ${ }^{13} \mathrm{C}$ NMR spectra of cross-linked PVA before and after the alkaline test, (b) FT-IR spectra of PVA, PCAD, cross-linked PVA before and after the alkaline test.

To confirm the stability of cross-linked PVA in the alkaline test, the solid-state ${ }^{13} \mathrm{C}$ NMR and FT-IR of cross-linked PVA before and after the alkaline test were carried out. As shown in Figure $\mathbf{S 5}$ (a), after immersing in $6 \mathrm{moL} \mathrm{L}^{-1} \mathrm{NaOH}$ at $60{ }^{\circ} \mathrm{C}$ for $250 \mathrm{~h}$, the chemical shifts of cross-linked PVA hardly changed compared those of sample before alkaline resistance test. The chemical shifts at position 7 ( $\delta=97.8 \mathrm{ppm})$ and position 12 $(\delta=169.8 \mathrm{ppm})$ indicated that the acetal structure and phenylate structure was still existed, which showed that cross-linked PVA was stable during the alkaline resistance test. In addition, FT-IR spectra of PVA, PCAD, cross-linked PVA before and after the alkaline test were demonstrated in Figure S5 (b). The peak at $3360 \mathrm{~cm}^{-1}$ was mainly due to the stretching vibration of the $-\mathrm{OH}$ groups from PVA or the possibly bound water. ${ }^{2}$ The peak at $2915 \mathrm{~cm}^{-1}$ arise from the stretching of $-\mathrm{CH}_{2}$ and $-\mathrm{CH}$ groups. ${ }^{3} \mathrm{~A}$ strong absorption band near 1087 and $1013 \mathrm{~cm}^{-1}$ corresponded to the vibration of CO-C. ${ }^{4}$ Compared with the FT-IR spectra of cross-linked PVA before the alkaline test, the spectra of cross-linked PVA remained unchanged after the alkaline test, which further verified that the cross-linked PVA was stable in the alkaline test. 
Table S1. IEC, $W U, S R_{l}$, and $\mathrm{OH}^{-}$Conductivity of QPPO Casting Membranes.

\begin{tabular}{cccccc}
\hline Membrane & $\mathrm{IEC}^{a}\left(\mathrm{mmol} \mathrm{g}^{-1}\right)$ & $\mathrm{WU}^{b}(\%)$ & $\mathrm{SR}_{\mathrm{l}}^{b}(\%)$ & $\begin{array}{c}\sigma\left(\mathrm{OH}^{-}\right)^{b} \\
\left(\mathrm{mS} \mathrm{cm}^{-1}\right)\end{array}$ & Ref \\
\hline QPPO-25 & 1.41 & 22.3 & 5.7 & 20.1 & This work \\
QPPO-50 & 2.30 & 75 & 13.8 & 30.4 & This work \\
QPPO & 1.00 & 15.1 & 2.2 & 16.7 & 5 \\
BTMA-30 & 2.03 & 65.7 & 12 & 26.4 & 6 \\
PPO-QA-40 & 2.64 & 130 & 180 & 24 & 7 \\
QPPO-40 & 2.27 & 72.2 & 11.8 & 22.5 & 1 \\
QPPO-60 & 3.22 & 457.0 & 67.5 & 33.6 & 1 \\
\hline
\end{tabular}

${ }^{a}$ Determined by titration at room temperature; ${ }^{b}$ Measured at room temperature, in the $\mathrm{OH}^{-}$ form 
Table S2. IEC, $W U$, In-plane $S R_{l}$, Through-plane $S R_{h}, \mathrm{OH}^{-}$Conductivity and Tensile Strength of QPPO/PVA Composite Membranes.

\begin{tabular}{cccccc}
\hline Sample & $\begin{array}{c}\mathrm{IEC}^{a} \\
\left(\mathrm{mmol} \mathrm{g}^{-1}\right)\end{array}$ & $\begin{array}{c}\mathrm{WU}^{b} \\
(\mathrm{wt} \%)\end{array}$ & $\begin{array}{c}\mathrm{SR}_{\mathrm{l}}^{b} \\
(\%)\end{array}$ & $\begin{array}{c}\sigma\left(\mathrm{OH}^{-}\right)^{c} \\
\left(\mathrm{mS} \mathrm{cm}^{-1}\right)\end{array}$ & $\begin{array}{c}\text { Strength }^{d} \\
(\mathrm{MPa})\end{array}$ \\
\hline $20 \mathrm{wt} \%$ QPPO/PVA & 0.42 & 78.2 & 20.6 & 5.3 & 26.9 \\
$20 \mathrm{wt} \%$ QPPONF/PVA & 0.43 & 92.1 & 22.9 & 11.1 & 21.6 \\
\hline $30 \mathrm{wt} \%$ QPPO/PVA & 0.62 & 60.9 & 18.7 & 6.3 & 25.8 \\
$30 \mathrm{wt} \%$ QPPONF/PVA & 0.65 & 77.8 & 20.0 & 13.2 & 20.1 \\
\hline $40 \mathrm{wt} \%$ QPPO/PVA & 0.81 & 48.2 & 13.1 & 11.8 & 23.5 \\
$40 \mathrm{wt} \%$ QPPONF/PVA & 0.77 & 64.2 & 15.0 & 29.0 & 17.4 \\
\hline $50 \mathrm{wt} \%$ QPPO/PVA & 1.02 & 35.1 & 10.2 & 21.4 & 27.8 \\
$50 \mathrm{wt} \%$ QPPONF/PVA & 1.02 & 45.0 & 10.4 & 60.9 & 19.2 \\
\hline $60 \mathrm{wt} \%$ QPPO/PVA & 1.19 & 32.5 & 10.4 & 30.0 & 26.0 \\
$60 \mathrm{wt} \%$ QPPONF/PVA & 1.20 & 43.1 & 12.3 & 73.1 & 21.2 \\
\hline $70 \mathrm{wt} \%$ QPPO/PVA & 1.41 & 30.2 & 9.8 & 39.6 & 27.4 \\
$70 \mathrm{wt} \%$ QPPONF/PVA & 1.39 & 43.0 & 10.1 & 89.3 & 22.2 \\
\hline
\end{tabular}

${ }^{a}$ Determined by titration with the Mohr method at room temperature; ${ }^{b}$ Measured at room temperature in the $\mathrm{OH}^{-}$form; ${ }^{c} \mathrm{OH}^{-}$conductivity at $80{ }^{\circ} \mathrm{C} ;{ }^{d}$ Measured at room temperature in the $\mathrm{OH}^{-}$form, with fully hydrated membranes. 


\section{References}

1. Han, J.; Zhu, L.; Pan, J.; Zimudzi, T. J.; Wang, Y.; Peng, Y.; Hickner, M. A.; Zhuang, L., Elastic Long-Chain Multication Cross-Linked Anion Exchange Membranes. Macromolecules 2017, 50 , 3323-3332.

2. Guo, D.; Zhuo, Y. Z.; Lai, A. N.; Zhang, Q. G.; Zhu, A. M.; Liu, Q. L., Interpenetrating anion exchange membranes using poly(1-vinylimidazole) as bifunctional crosslinker for fuel cells. J. Membr. Sci. 2016, 518, 295-304.

3. Pandit, S.; Khilari, S.; Bera, K.; Pradhan, D.; Das, D., Application of PVA-PDDA polymer electrolyte composite anion exchange membrane separator for improved bioelectricity production in a single chambered microbial fuel cell. Chem. Eng. J. 2014, 257, 138-147.

4. Das, K.; Ray, D.; Bandyopadhyay, N. R.; Gupta, A.; Sengupta, S.; Sahoo, S.; Mohanty, A.; Misra, M., Preparation and Characterization of Cross-Linked Starch/Poly(vinyl alcohol) Green Films with Low Moisture Absorption. Ind. Eng. Chem. Res. 2010, 49, 2176-2185.

5. Cheng, X.; Wang, J.; Liao, Y.; Li, C.; Wei, Z., Enhanced Conductivity of AnionExchange Membrane by Incorporation of Quaternized Cellulose Nanocrystal. ACS Appl. Mater. Interface 2018, 10, 23774-23782.

6. Liu, L.; Chu, X.; Liao, J.; Huang, Y.; Li, Y.; Ge, Z.; Hickner, M. A.; Li, N., Tuning the properties of poly(2,6-dimethyl-1,4-phenylene oxide) anion exchange membranes and their performance in $\mathrm{H} 2 / \mathrm{O} 2$ fuel cells. Energy Environ. Sci. 2018, 11, 435-446.

7. Li, N.; Leng, Y.; Hickner, M. A.; Wang, C.-Y., Highly Stable, Anion Conductive, Comb-Shaped Copolymers for Alkaline Fuel Cells. J. Am. Chem. Soc. 2013, 135, 10124-10133. 